\title{
ACERCA DE LA 1 SEMANA MUNDIAL DE LAS INMUNIZACIONES
}

\section{FIRST WORLD INMUNIZATION WEEK}

\author{
Guido Bendezu-Quispe ${ }^{1,2, a}$, Lucero Choque Guevara ${ }^{3, b}$ \\ 1. Facultad de Medicina Humana, Universidad Nacional San Luis Gonzaga. Ica, Perú \\ 2. Sociedad Científica de Estudiantes de Medicina de Ica \\ 3. Facultad de Enfermería, Universidad Nacional San Luis Gonzaga. Ica, Perú \\ a. Estudiante de Medicina Humana \\ b. Estudiante de enfermería
}

Señor Editor:

La inmunización constituye una de las herramientas costoefectivas más importantes con las que cuenta el Sistema de Salud. Permite disminuir la morbilidad, discapacidad y mortalidad asociada a enfermedades infecciosas prevenibles a través de vacunas. En el 2012 se conmemora el $10^{\circ}$ Aniversario de la Semana de Vacunación de las Américas, referente en temas de inmunización a nivel global, con el establecimiento de la Semana Mundial de Inmunización (21-28 abril) que busca ser un marco general de los esfuerzos en conjunto que se realizan sobre el tema (1)

Desde que Jenner publicara sus resultados en 1798 en Variolae Vaccinae acerca del estudio sobre el inóculo realizado al niño James Phipps de la linfa de una pústula de viruela obtenida de la ordeñadora Sara Nelmes y la profilaxis que adquirió ante la enfermedad, se desató un interés mundial en poder prevenir el desarrollo de otras enfermedades infecciosas por medio de la vacunación ${ }^{(2)}$.

La Organización Mundial de la Salud (OMS) estima que 2,5 millones de niños menores de cinco años mueren cada año por enfermedades prevenibles con vacunas. Esto se traduce en más de 600 muertes de infantes por día. Es por ello que la vacunación es pieza fundamental para el logro de los Objetivos de Desarrollo del Milenio que buscan disminuir la mortalidad en menores de 5 años ${ }^{(3)}$.

La OMS y el Fondo de las Naciones Unidas para la Infancia (UNICEF) en colaboración con otros asociados establecieron la Visión y Estrategia Mundial de Inmunización (GIVS) que es el primer marco estratégico a 10 años (2006-2015) para responder a los retos de la inmunización y combatir las enfermedades prevenibles con vacunas, busca el aumento del alcance de la inmunización mundial para estas enfermedades comunes, señalando que al 2010 en todos los países esta debería llegar al 90\% de la población ${ }^{(3)}$.

En las Américas, los programas de inmunizaciones han sido realmente exitosos, ejemplos para el resto del mundo al ser los primeros en erradicar enfermedades tales como la Viruela y Poliomielitis. Respecto al Sarampión, en el Perú, desde marzo del 2000 no se confirma un solo caso ${ }^{(4)}$. El tétanos neonatal no es en la actualidad un problema de salud pública desde que en 1992 se iniciara la inmunización de mujeres en edad fértil, presentándose solo 1 caso durante el año 2009 teniendo en cuenta que durante el año 1995 el número de casos fuera de $94^{(5)}$.

En el país, el Programa Nacional de Inmunizaciones existió desde 1972 hasta el año 2001, año en que la actividad se incorporó al programa de atención integral de salud del niño. La Estrategia Sanitaria Nacional de Inmunizaciones fue creada el 27 de julio del 2004 con la finalidad de señalar la prioridad política, técnica y administrativa que el pueblo y Gobierno Peruano reconocen en las inmunizaciones.

Respecto a la cobertura de las inmunizaciones, al año 1970, los esquemas de vacunación de los países incluían cuatro vacunas que protegían contra seis enfermedades (formas graves de tuberculosis, difteria, tos ferina, tétanos, poliomielitis y sarampión teniendo una cobertura no mayor al $10 \%{ }^{(3)}$. En nuestro país, ya para el año 2009 , un estimado de la OMS/UNICEF muestra que la cobertura actual es superior al $90 \%$ (Tabla 1) esto debido al impacto de los programas de inmunización nacional así como a nivel continente Americano.

El establecimiento de la Semana Mundial de las inmunizaciones llama a la reflexión del impacto que ha tenido esta actividad en la salud de la población mundial. Para el periodo 2005-2007 se ha alcanzado la vacunación de más de 100 millones de niños, cifra nunca antes vista, además que los beneficios de las inmunizaciones están llegando a otros grupos de riesgo de enfermedades inmunoprevenibles como los adolescentes y los adultos, protegiéndolos contra enfermedades que ponen en peligro la vida como la gripe, la meningitis y ciertos cánceres que aparecen en la edad adulta ${ }^{(3)}$.

A pesar de estos logros, en el 2007 se evidencio que casi el $20 \%$ de los que nacen cada año, quedaron sin recibir todas las inmunizaciones, esto es 24 millones de niños sin protección ${ }^{(3)}$. La disminución de carga de morbilidad y mortalidad en el país, debida a las inmunizaciones, debe servir de estimulo para disminuir las brechas de cobertura aún existentes y fortalecer en el tiempo los logros obtenidos de la aplicación de la misma en nuestra población. 
Tabla 1: Razón de cobertura Nacional - Perú 2009 (Estimado OMS/UNICEF)

\begin{tabular}{ccccccccc}
\hline & $\mathbf{2 0 0 9}$ & $\mathbf{2 0 0 8}$ & $\mathbf{2 0 0 7}$ & $\mathbf{2 0 0 6}$ & $\mathbf{2 0 0 5}$ & $\mathbf{2 0 0 0}$ & $\mathbf{1 9 9 0}$ & $\mathbf{1 9 8 0}$ \\
\cline { 2 - 8 } BCG & $99 \%$ & $99 \%$ & $99 \%$ & $99 \%$ & $93 \%$ & $93 \%$ & $83 \%$ & $57 \%$ \\
DTP1 & $98 \%$ & $98 \%$ & $98 \%$ & $98 \%$ & $98 \%$ & $98 \%$ & $89 \%$ & $39 \%$ \\
DTP3 & $93 \%$ & $99 \%$ & $97 \%$ & $94 \%$ & $89 \%$ & $98 \%$ & $72 \%$ & $16 \%$ \\
HepB3 & $93 \%$ & $99 \%$ & $93 \%$ & $94 \%$ & $89 \%$ & $\ldots$ & $\ldots$ & $\ldots$ \\
Hib3 & $93 \%$ & $99 \%$ & $93 \%$ & $94 \%$ & $89 \%$ & $38 \%$ & $\ldots$ & $\ldots$ \\
\hline
\end{tabular}

BCG: Bacilo de Calmette-Guérin; DTP1: $1^{\underline{a}}$ dosis de toxoide diftérico y tetánico con vacuna para pertusis;

DTP3: $3^{\underline{a}}$ dosis de toxoide diftérico y tetánico con vacuna para pertusis; HepB3: $3^{\underline{a}}$ dosis de vacuna hepatitis

B; Hib3: $3^{\text {a }}$ dosis vacuna para Haemophilus influenzae tipo B; MCV: Vacuna conjugada contra meningococo;

Pol3: $3^{\underline{a}}$ dosis vacuna de la polio; PAB: Protección contra tétanos neonatal.

\section{REFERENCIAS BIBLIOGRÁFICAS}

1. Global Health Magazine (Sitio de Internet).

Disponible en:

http://www.globalhealthmagazine.com/cover stories Ivaccination week goes global. Acceso el 2 de abril del 2012

2. Berdasquera D, Cruz G., Suárez C. La vacunación. Antecedentes históricos en el mundo. Rev Cubana Med Gen Integr 2000;16(4):375-8

3. OMS, UNICEF, Banco Mundial. Vacunas e inmunización: situación mundial, tercera edición. Ginebra, Organización Mundial de la Salud, 2010.
4. OPS. Introducción e implementación de nuevas vacunas. Guía práctica (Sitio de Internet). Disponible en http://www.paho.org/spanish/ad/fch/im/guiapractica intro nuevasvacunas.pdf. Acceso el 3 de abril del 2012.

5. MINSA, DGE. ASIS Perú 2010. Lima, Ministerio de Salud del Perú,2010

\section{Correspondencia:}

Guido Bendezú Quispe

Correo electrónico:

g.bendezu-quispe@hotmail.com 